\title{
Different Communication Protocols for Wireless Sensor Networks: A Review
}

\author{
Meena Ahlawat ${ }^{1}$, Ankita Mittal $^{2}$ \\ M.Tech student, ECE Department, GGGI, Ambala, India ${ }^{1}$ \\ A.P, ECE Department, GGGI, Ambala, India ${ }^{2}$
}

\begin{abstract}
Recent development in the area of micro-sensor devices have accelerated the advances in the sensor networks field leading to many new protocols specifically designed for wireless sensors networks(WSNs).wireless sensors networks with hundreds to thousands of sensor nodes can gather information from an unattended location and transmit the gathered data to a particular users, depending on the applications. These sensor nodes have some constraints due to their limited energy, storage capacity and computing power. Data are routing from one node to other using different routing protocols. There this paper gives brief idea about different protocols in wireless sensor networks (like LEACH, SEP, HRC etc.) also included comparison of these protocols.
\end{abstract}

Keywords: WSN, protocols, routing, LEACH, SEP, HRC.

\section{INTRODUCTION}

Wireless sensors networks are networks of tiny, battery powered sensor nodes with limited on -board processing storage and radio capabilities. Nodes sense and send their reports towards a processing centre which is called "sink". The design of protocols and applications for such network has to be energy aware in order to prolong the lifetime of the network, because the replacement of the embedded batteries is a very difficult process once these nodes have been deployed. As shown in figure 1 the regular nodes sense the field, generate the data, and send them to associated nodes.[2,3] Then the after performing some processes transmit them to the BS in a multi-hop approach. Eventually the user receives the data from the BS through the Internet.

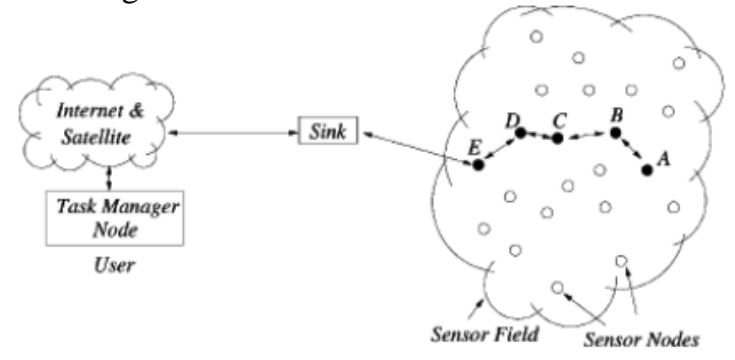

Figure 1: Wireless Sensor Network

\section{PROTOCOL PROPOSED FOR WIRELESS NETWORKS}

Wireless sensor networks (WSNs) are composed of a huge number of sensor nodes. There are many applications for WSNs and depending on the application, different types of sensors are used, such as sensors measuring moisture, temperature, pressure and movement. WSNs have themselves characteristics that make them different from other types of networks. One for example is that the applicability of the networks is related to energy supply of the nodes, so energy conservation is one of the most important challenges in these networks. Different types of protocols for WSN are following:

\section{A. Direct transmission protocols}

Using a direct communication protocol, each sensor sends its data directly to the base station. If the base station is far away from the nodes, direct communication will require a large amount of transmit power from each node. This will quickly drain the battery of the nodes and reduce the system lifetime. How- ever, the only receptions in this protocol occur at the base station, so if either the base station is close to the nodes, or the energy required receiving data is large, this may be an acceptable method of communication.

\section{B. minimum transfer energy protocols}

In these protocols, nodes act as routers for other nodes' data in addition to sensing the environment. These protocols differ in the way the routes are chosen. Some of these protocols only consider the energy of the transmitter and neglect the energy dissipation of the receivers in determining the routes. Depending on the real time costs of the transmit amplifier and the radio electronics, the total energy expended in the system might actually be greater using MTE routing than direct transmission to the base station. Figure 2 shows the number of sensors that remain alive after each round for direct transmission and MTE routing.

This shows that, as predicted by our analysis above, when transmission energy is on the same order as receive energy, which occurs when transmission distance is short and/or the radio electronics energy is high, direct transmission is more energy-efficient on a global scale than MTE routing. It is clear that in MTE routing, the nodes closest to the base station will be used to route a large number of data messages to the base station.

Thus these nodes will die out quickly, causing the energy required to get the remaining data to the base station to increase and more nodes to die. 


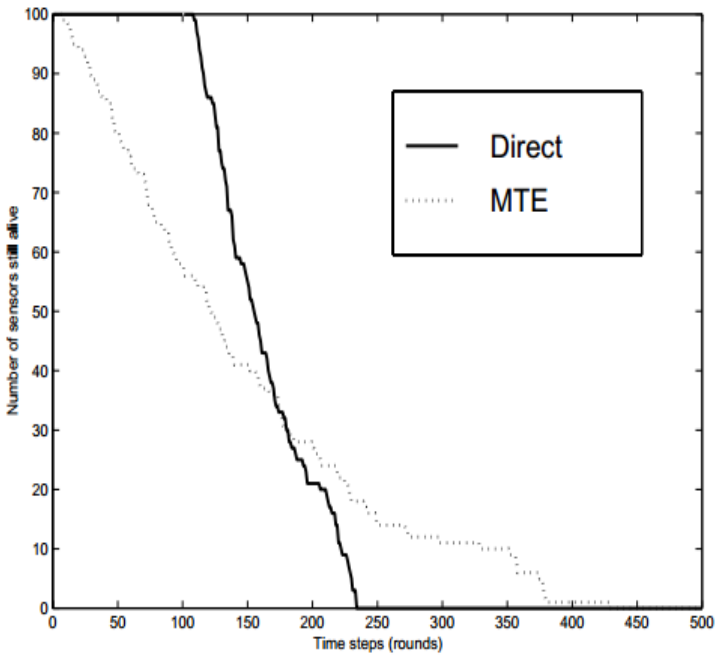

Figure 2: Comparison of Lifetime Between Direct Transmission and MTE. [2]

This will create a cascading effect that will shorten system lifetime. In addition, as nodes close to the base station die, that area of the environment is no longer being monitored. To prove this point, this plot shows that nodes die out quicker using MTE routing than direct transmission.
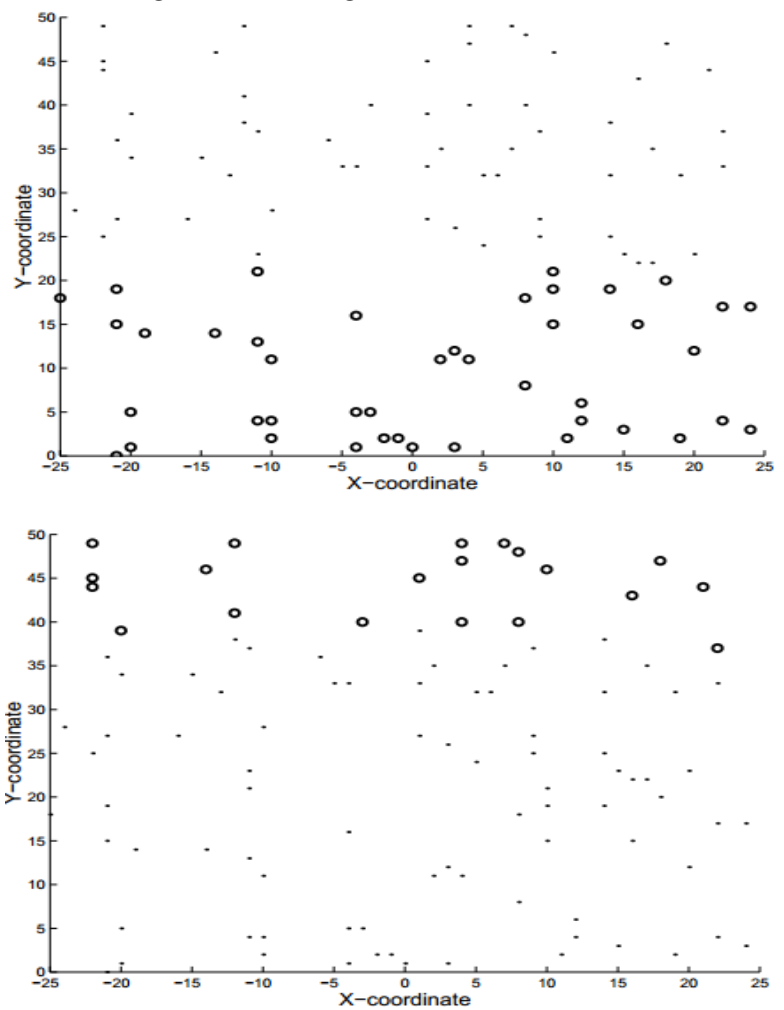

Figure 3: Alive and Dead Node in Direct Transmission and MTE [2].

Figure 3 shows that nodes closest to the base station are the ones to die out first for MTE routing, whereas nodes furthest from the base station are the ones to die out first for direct transmission. This is as expected, since the nodes close to the base station are the ones most used as "routers" for other sensors' data in MTE routing, and the nodes furthest from the base station have the largest transmit energy in direct communication.

\section{C.clustering protocol}

A final conventional protocol for wireless networks is clustering, where nodes are organized into clusters that communicate with a local base station, and these local base stations transmit the data to the global base station, where it is accessed by the end-user. This greatly reduces the distance nodes need to transmit their data, as typically the local base station is close to all the nodes in the cluster. Thus, clustering appears to be an energy-efficient communication protocol. However, the local base station is assumed to be a high-energy node; if the base station is an energy-constrained node, it would die quickly, as it is being heavily utilized. Thus, conventional clustering would perform poorly for our model of micro sensor networks. The Near Term Digital Radio (NTDR) project $[12,16]$, an army-sponsored program.

\section{LEACH: Low-Energy Adaptive Clustering Hierarchy} $\mathrm{LEACH}$ is a self-organizing, adaptive clustering protocol that uses randomization to distribute the energy load evenly among the sensors in the network. In LEACH, the nodes organize themselves into local clusters, with one node acting as the local base station or cluster head. LEACH includes randomized rotation of the high-energy cluster-head position such that it rotates among the various sensors in order to not drain the battery of a single sensor. LEACH performs local data fusion to "compress" the amount of data being sent from the clusters to the base station, further reducing energy dissipation and enhancing system lifetime.[1,6] Figure 4 below shows dynamic clustering.

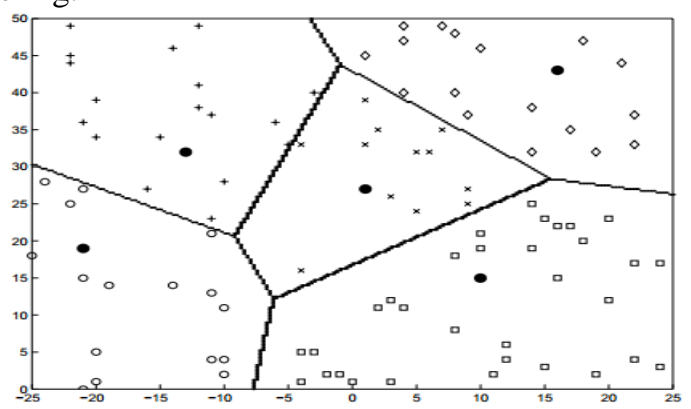

Figure 4: Dynamic Clustering [5]

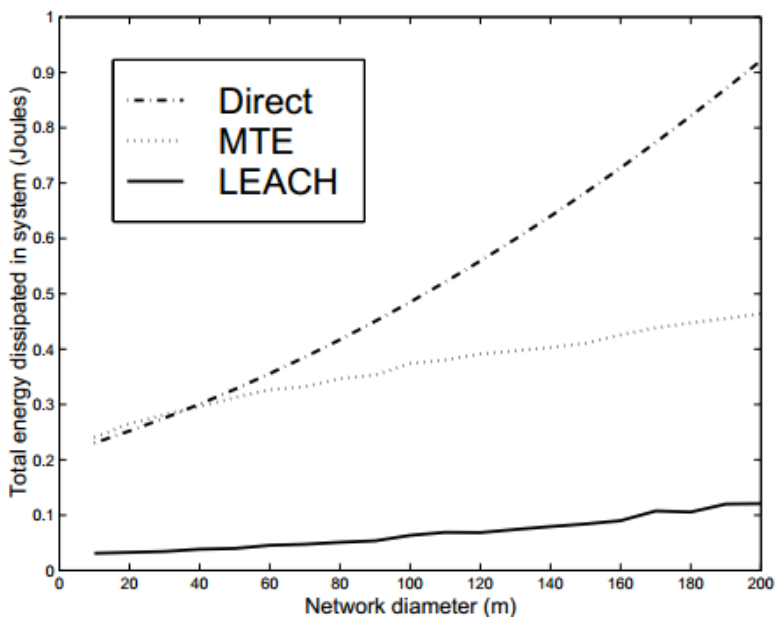

Figure 5: Energy Dissipated using Direct Transmission, MTE and LEACH.[7,1] 
Figure 5 shows that LEACH achieves between $7 x$ and $8 x$ reduction in energy compared with direct communication and between $4 x$ and $8 x$ reduction in energy compared with MTE routing.

\section{E. Stable Election Protocol}

Stable Election Protocol (SEP) is proposed based on weighted election probabilities of each node to become cluster-head (CHs) according to their respective energy. This approach ensures that the cluster head election is randomly selected and distributed based on the fraction of energy of each node assuring a uniform use of the nodes energy. SEP also considered two types of nodes and two level hierarchies.

we have analysed a three level hierarchical clustered heterogeneous sensor network and considered three types of sensor nodes having different initial energy namely advanced, moderate and normal nodes.[5] Advanced and moderate nodes have more energy supply, longer transmission range, higher data rate than the normal nodes. So, the advanced and moderate nodes have higher chances to become cluster heads at a particular round compare to a normal node which extensively prolong the sensor network to operate.

\section{F. Hierarchical Cluster-Based Routing (HCR) Protocol} In HCR nodes self organize into clusters and each cluster is managed by a set of associates called head-set. Using round-robin technique, each associate acts as a cluster head $(\mathrm{CH})$.[3] The sensor nodes transmit data to their cluster heads, which transmit the aggregated data to the base station. Moreover, the energy-efficient clusters are retained for a longer period of time; the energy-efficient clusters are identified using heuristics-based approach.

\section{G. Genetic Algorithm}

A genetic algorithm (GA) is used to create energy efficient clusters for data dissemination in wireless sensor networks. A GA is used at the base station, which provides energy efficient solutions to the optimizer. This provides the base station with the ability to determine the best cluster formation that will give minimum energy consumption during run time. The base station analyses the current network condition and applies the GA after every iteration. The optimizer at the base station selects the best solution based on the acquired knowledge through the GA fitness function. [9]The proposed fitness function is based on parameters such as energy consumption, number of clusters, cluster size, direct distance to sink, and cluster distance. Upon completion of iteration, the optimizer improves its decisions by receiving feedback, which is then used to adjust the weights of the parameters of the fitness function for the next iteration.

The BS uses a GA to create energy-efficient clusters for a given number of transmissions. The node is represented as a bit of a chromosome. The head and member nodes are represented as $1 \mathrm{~s}$ and $0 \mathrm{~s}$, respectively. A population consists of several chromosomes and the best chromosome is used to generate the next population. The simulation results indicate that using GA-based hierarchical clusters increase the network life time. In future, the cross layer optimization using query and routing strategies can be investigated.

TABLE I

COMPARISON OF PROTOCOLS

\begin{tabular}{|c|c|c|}
\hline Sr.No. & protocols & Comparisons \\
\hline 1. & $\begin{array}{l}\text { Direct } \\
\text { transmission }\end{array}$ & $\begin{array}{l}\text { 1. In this protocol sensor node } \\
\text { transmit data directly to sink. } \\
\text { 2. Nodes far from sink die } \\
\text { quickly. } \\
\text { 3. Not energy efficient protocol. }\end{array}$ \\
\hline 2. & MTE & $\begin{array}{l}\text { 1. In these protocols, nodes act as } \\
\text { routers for other nodes. } \\
\text { 2. Nodes near to sink die first. } \\
\text { 3. Require more energy than DT. }\end{array}$ \\
\hline 3. & Clustering & $\begin{array}{l}\text { 1.one head for some nodes } \\
\text { 2. Transmission through cluster } \\
\text { head. } \\
\text { 3. Work poorly because head die } \\
\text { quickly. }\end{array}$ \\
\hline 4. & LEACH & $\begin{array}{l}\text { 1. The nodes organize themselves } \\
\text { into local clusters. } \\
\text { 2. Includes randomized rotation } \\
\text { of the high-energy cluster-head } \\
\text { position so not drain the battery of } \\
\text { single node. } \\
\text { 3. Give transmission energy } \\
\text { homogenously. }\end{array}$ \\
\hline 5. & SEP & $\begin{array}{l}\text { 1. Increase system lifetime with } \\
\text { increase stability. } \\
\text { 2. Transmission energy } \\
\text { heterogeneous. }\end{array}$ \\
\hline 6 & HCR & $\begin{array}{c}\text { Based on hierarchy of cluster } \\
\text { head and sink. }\end{array}$ \\
\hline 7 & GA & $\begin{array}{l}\text { A genetic algorithm (GA) is used } \\
\text { to create energy efficient clusters } \\
\text { for data dissemination in wireless } \\
\text { sensor networks. }\end{array}$ \\
\hline
\end{tabular}

\section{III.CONCLUSION}

WSNs are composed of hundreds or thousands of sensor nodes that are randomly dispersed in harsh environments. Due to the limited access to the nodes, self-organization and topology management are essential characteristics in these networks. More importantly, sensor nodes are drastically energy constrained so that preserving the energy is one of the most important challenges in WSNs. Researchers have proposed many algorithms to solve these challenges. In this paper, we presented a comprehensive and state-of-the-art.

In this paper we presented a comprehensive survey on the different approaches. First, we explained the problem in wireless sensor networks and its applications, objectives and characteristics. Then we reviewed and classified the most important approaches and their extensions. All methods generally try to improve the lifespan of the network the main design challenge in these methods is basically different. 


\section{REFERENCES}

[1] Akyildiz I, Su W, Sankarasubramaniam Y, Cayirci E. "A survey on sensor networks" IEEE Commun Mag, 40:102-14, 2002

[2] Al-Karaki JN, Kamal AE. "Routing techniques in wireless sensor networks: a survey" IEEE Wirel Commun 11:6-28.S, 2004.

[3] Anastasi G, Conti M, Di Francesco M, Passarella A "Energy conservation in wireless sensor networks: a survey" Ad Hoc Netw 7:537-68, 2007.

[4] Baek J, An SK, Fisher P. "Dynamic cluster header selection and conditional re-clustering for wireless sensor networks." IEEE Trans Consum Electron 56:2249-57, 2010.

[5] Banerjee S, Khuller S. "A clustering scheme for hierarchical control in multi-hop wireless networks" IEEE conference on communications (INFOCOM), vol. 2, Anchorage, Alaska, USAl; p. 1028-37,2001.

[6] Cheng C, Tse C, Lau F. "A clustering algorithm for wireless sensor networks based on social insect colonies" IEEE Sens J 11:711-21, 2011 .

[7] Dahnil DP, Singh YP, Ho C-K. "Topology-controlled adaptive clustering for uniformity and increased lifetime in wireless sensor networks" IET Wirel Sens Syst 2:318-27,2012.

[8] Kang Sang H, Nguyen Thinh. "Distance based thresholds for cluster head selection in wireless sensor networks" IEEE Commun Lett 16(9):1396-9.2012.

[9] Kaur T, Baek J. "A strategic deployment and cluster-header selection for wireless sensor networks" IEEE Trans Consum Electron, 55:1890-7, 2009. 